\title{
SOME THEOREMS ON GENERALIZED POLARS WITH ARBITRARY WEIGHT
}

\section{NEYAMAT ZAHEER}

Mathematics Department

King Saud University

Riyadh, Saudi Arabia

and

\section{AIJAZ A. KHAN}

Mathematics Department

Aligarh Muslim University

Aligarh, India

(Received September 2, 1986)

ABSTRACT. The present paper, which is a continuation of our earlier work in Annali di Mathematica [1] and Journal Math. Seminar [2] (Er EuOEPIA), University of Athens, Greece, deals with the problem of determining sufficiency conditions for the nonvanishing of generalized polars (with a vanishing or nonvanishing weight) of the product of abstract homogeneous polynomials in the general case when the factor polynomials have been preassigned independent locations for their respective nul1sets. Our main theorems here fully answer this general problem and include in them, as special cases, all the results on the topic known to date and established by Khan, Marden and Zaheer (see Pacific J. Math. 74 (1978), 2, pp. 535-557, and the papers cited above). Besides, one of the main theorems leads to an improved version of Marden's general theorem on critical points of rational functions of the form $f_{1} f_{2} \ldots f_{p} / f_{p+1} \ldots f_{q}, f_{i}$ being complex-valued polynomials of degree $n_{i}$.

KEY WORDS AND PHRASES. Generalized circular regions, circular cones, generalized polars, abstract homogeneous polynomials.

1980 AMS SUBJECT CLASSIFICATION CODE. Primary 30Cl5, Secondary 12D10.

\section{INTRODUCTION.}

A few years ago, the concept of generalized polars of the product of abstract homogeneous polynomials (a.h.p.) was introduced by Marden [3] while in his attempt to generalize to vector spaces a theorem due to Bôcher [4]. His formulation involves the use of hermitian cones [5], a concept which was first used by Hörmander [6] in obtaining a vector space analogue of Laguerre's theorem on polar-derivatives [7] and, later, employed by Marden [3], [8], in the theory of composite a.h.p.'s. In all these areas the role of the class of hermitian cones has been replaced by a strictly larger class of the so-called circular cones. This was successfully done by Zaheer [9], [10], [11] and [5] in presenting a more general and compact theory which incorporates into it the various independent studies made by Hormander, Marden and Zervos. A complete account of the work to date on generalized polars, which fall in the category of composite a.h.p.'s in the wider sense of the definition of the latter now in use (cf. [5], [12], [13], [14]) can be found in the papers due to Marden [3], Zaheer [5], and 
the authors [1], [2]. Generalized polars with a vanishing weight as well as the ones with a non-vanishing weight have been considered in the first two papers, while the third (resp. the fourth) deals exclusively with the ones having a vanishing (resp. a non-vanishing) weight. But all have a common feature that the factor polynomials involved in the generalized polar of the product have been divided into two or three groups, each of which is preassigned a circular cone containing the nul1-sets of all polynomials belonging to that group. Our aim here is to consider generalized polars with a vanishing or a non-vanishing weight where, in general, no two factor polynomials are necessarily required to have the same circular cone in which their null-sets must lie. In fact, we take up the general problem of determining sufficiency conditions for the non-vanishing of generalized polar (with a vanishing or a non-vanishing weight) where the factor polynomials have been preassigned mutually independent locations for their respective null-sets. Our main theorems fully answer this general problem and include in them, as special cases, all the corresponding results on the topic known to date and established in Marden [3], Zaheer [5] and the authors [1], [2]. One of the main theorems of this paper leads to a slightly improved form of Marden's general theorem on critical points of rational functions [7].

\section{PRELIMINARIES.}

Throughout we let $\mathrm{E}$ and $\mathrm{V}$ denote vector spaces over a field $\mathrm{K}$ of characteristic zero. A mapping $\mathrm{P}: \mathrm{E} \rightarrow \mathrm{V}$ is called (cf. [6], [15], [16], and [9]) a vector-valued $a . h . p$. of degree $\mathrm{n}$ if (for each $\mathrm{x}, \mathrm{y} \varepsilon \mathrm{E}$ )

$$
P(s x+t y)=\sum_{k=0}^{n} A_{k}(x, y) s^{k} t^{n-k} \quad \forall s, t \varepsilon k,
$$

where the coefficients $A_{k}(x, y) \varepsilon V$ depend only on $x$ and $y$. We shall call $P$ an a. h. p. (resp. an algebra-valued a. h. p.) if $\mathrm{V}$ is taken as $\mathrm{K}$ (resp. an algebra). We denote by $P_{n}^{*}$ the class of all vector-valued a.h.p.'s of degree $n$ from $E$ to $V$ (even if $V$ is an algebra) and by $P_{n}$ the class of all a.h.p's of degree $n$ from $E$ to K. The nth polar of $P$ is the unique symmetric $n-1$ inear form $P\left(x_{1}, x_{2}, \ldots, x_{n}\right)$ from $E^{n}$ to $V$ such that $P(x, x, \ldots, x)=P(x)$ for all $x \in E$ (Hormander [6] and Hille and Phillips [15] for its existence and uniqueness). The kth polar of $P$, for given $x_{1}, x_{2}, \ldots, x_{k}$ in $E$, is defined by

$$
P\left(x_{1}, x_{2}, \ldots, x_{k}, x\right)=P\left(x_{1}, \ldots, x_{k}, x, \ldots, x\right) \text {. }
$$

The following material is borrowed from Zaheer [17], [1] and [2] . Given $\mathrm{m}_{k} \varepsilon \mathrm{K}$ and $\mathrm{P}_{\mathrm{k}} \varepsilon \mathbf{P}_{\mathrm{n}_{\mathrm{k}}}^{*}(\mathrm{k}=1,2, \ldots, \mathrm{q})$, we write

$$
\begin{aligned}
Q(x) & =P_{1}(x) P_{2}(x) \ldots P_{q}(x), \\
Q_{k}(x) & =P_{1}(x) \ldots P_{k-1}(x) P_{k+1}(x) \ldots P_{q}(x),
\end{aligned}
$$

and

$$
\Phi\left(Q ; x_{1}, x\right)=\sum_{k=1}^{q} m_{k} Q_{k}(x) P_{k}\left(x_{1}, x\right) \quad \forall x, x_{1} \varepsilon E,
$$


and define $P\left(Q ; x_{1}, x\right)$ as an algebra-valued generalized polar of the product $Q(x)$ [5]. The scalar $\sum_{k=1}^{\mathrm{q}} \mathrm{m}_{\mathrm{k}}$ is called its weight. The Term 'generalized polar' will be used in special reference to the case when $V=K$, so as to conform with the existing terminology [5]. As in Hille and Phillips [15], if $n=n_{1}+n_{2}+\ldots+n_{q}$, we recall that $Q \in P_{n}^{*}, Q_{k} \in P_{n-n_{k}}^{*}$ and $P_{k}\left(x_{1}, x\right)$ is an algebra-valued a.h.p. of degree $n_{k}-1$ in $x$ and of degree 1 in $x_{1}, 1 \leq k \leq q$. Therefore, $D\left(Q: x_{1}, x\right)$ is an algebra-valued a.h.p. of degree $n-1$ in $x$ and of degree 1 in $x_{1}$.

Given a nontrivial scalar homomorphism $L: V \rightarrow K$ [18] and [1] and a polynomial $P \in P_{n}^{*}$, we define the mapping $L P: E \rightarrow K$ by

$$
(L P)(x)=L(P(x)) \quad \forall x \in E \text {. }
$$

Obviously, LP $\varepsilon \mathbf{P}_{\mathrm{n}}$. In the notations of (2.1) and (2.2) the product of the polynomials ${ }^{L P_{k}} \in P_{n_{k}}$ is given by $L Q$ and the corresponding partial product (LQ)k (achieved by deleting the kih factor in the expression for LQ) is given by $L_{k^{*}} T^{*}$ is immediately leads to the following

REMARK 2.1. The algebra-valued generalized polar $\$\left(Q ; x_{1}, x\right)$ of the product $Q(x)$ and the generalized polar $Q\left(L Q ; x_{1}, x\right)$ of the corresponding product (LQ)( $\left.x\right)$, with the same $m_{k}$ 's satisfy the relation

$$
L\left(\$\left(Q ; x_{1}, x\right)\right)=\$\left(L Q ; x_{1}, x\right)
$$

for every nontrivial scalar homomorphism $\mathrm{L}$ on $\mathrm{V}$.

If $K$ is an algebraically closed field of characteristic zero, then we know [19] and [20] that $K$ must contain a maximal ordered subfield $K_{0}$ such that $K=K_{0}(i)$, where $-i^{2}$ is the unity element in $K$. For any element $z=a+i b \varepsilon K\left(a, b \varepsilon K_{0}\right.$ ) we define $\bar{z}=a-i b, \operatorname{Re}(z)=(z+\bar{z}) / 2$, and $|z|=+\left(a^{2}+b^{2}\right)^{1 / 2}$ in analogy with the complex plane. We denote by $K_{\omega}$ the projective field [5] and [21] achieved by adjoining to $\mathrm{K}$ an element $\omega$ having the properties of infinity, and, by $D\left(K_{\omega}\right)$, the class of all generalized circular regions (g.c.r.) of $\mathrm{K}_{\omega}$. The notions of $\mathrm{K}_{0}-$ convex subsets of $K$ and of $D\left(K_{\omega}\right)$ are due to Zervos [21], but the definitions and a brief account of relevant details can be found in [5]. For special emphasis in the field $\mathbf{C}$ of complex numbers, we state the following characterization of $\mathrm{D}\left(\mathbf{c}_{\omega}\right): T_{\text {The }}$ nontrivial g.c.r.'s of $\mathbf{c}_{\omega}$ are the open interior (or exterior) of circles or the open half-planes, adjoined with a connected subset (possibly empty) of their boundary. The g.c.r.'s of $\mathbf{c}_{\omega}$, with all or no boundary points included, are termed as (classical) circular regions (c. r.) of $\mathbf{C}_{\omega}$.

In vector space $E$ over an algeraically closed field $K$ of characteristic zero, the terms 'nucleus', 'circular mapping' and 'circular cone' are due to Zaheer [5]. Given a nucleus $N$ of $E^{2}$ and a circular mapping $G: N \rightarrow D\left(K_{\omega}\right)$, we define the circular cone $\mathrm{E}_{\mathrm{o}}(\mathrm{N}, \mathrm{G})$ by

$$
E_{0}(N, G)=\bigcup_{(x, y) \varepsilon N} T_{G}(x, y),
$$


where

$$
T_{G}(x, y)=\{s x+2 y \neq 0 \mid s, t \in K ; s / t \in G(x, y)\}
$$

REMARK 2.2. (I) [1]. If $G$ is a mapping from $\mathrm{N}$ into the class of all subsets of $K_{\omega}$ (so that $G(x, y)$ may not necessarily be a $g \cdot c_{\cdot} r_{\bullet}$ ), the resulting set $E_{0}(N, G)$ will be termed only a cone in $\mathrm{E}$.

(II) If $\operatorname{dim} E=2$, then [10] every circular cone $E_{0}(N, G)$ is of the form

$$
E_{0}(N, G)=\left\{s x_{0}+t y_{0} \neq 0 \mid s, t \varepsilon K ; s / t \varepsilon A\right\}
$$

for some $A \varepsilon D\left(K_{\omega}\right)$, where $x_{0}, y_{o}$ are any two linearly independent elements of $E$, with $N=\left\{\left(x_{0}, y_{0}\right)\right\}$ and $G\left(x_{0}, y_{0}\right)=A$.

(III) We remark [5] that any two (and, hence, any finite number of) circular cones can always be expressed relative to an arbitrarily selected common nucleus.

3. THE CENTRAL THEOREM.

Unless mentioned otherwise, $K$ denotes an algebraically closed field of characteristic zero, $E$ a vector space over $K$, and $V$ an algebra with identily over $K$. The field of complex numbers is denoted by $c$. We denote by $L[x, y]$ the the subspace of $E$ generated by elements $x$ and $y$ of $E$, and by $L^{2}[x, y]$ the set product $L[x, y] \times L[x, y]$ (i.e. the set of all ordered pairs of elements from $\mathcal{L}[\mathrm{x}, \mathrm{y}])$.

In this section we establish the central theorem of this paper, which gives sufficiency conditions for the non-vanishing of generalized polars having a vanishing or a non-vanishing weight and which answers the general problem mentioned in the introduction. Apart from deducing the main theorems of the authors proved earlier in [1] and [2] the present theorem applies in the complex plane to yield an improved form of a general theorem due to Marden [7]. In the following theorem we take, without loss of generality (cf. Remark 2.2 (III)), circular cones with a common nucleus. Consistently, we shall denote by $z_{p}(x, y)$ the nulz-set of an a.h.p. p (with respect to given elements $x, y \in E$ ), defined by

$$
Z_{P}(x, y)=\{s x+t y \neq o \mid s, t \varepsilon K ; P(s x+t y)=o\} \text {. }
$$

THEOREM 3.1. For $\mathrm{k}=1,2, \ldots, \mathrm{q}$, let $\mathrm{P}_{\mathrm{k}} \varepsilon \mathrm{P}_{\mathrm{n}_{\mathrm{k}}}$ and $\mathrm{E}_{\mathrm{o}}^{(\mathrm{k})} \equiv \mathrm{E}_{\mathrm{o}}\left(\mathrm{N}, \mathrm{G}_{\mathrm{k}}\right)$ be circular cones in $\mathrm{E}$ such that $\mathrm{Z}_{\mathrm{P}_{\mathrm{k}}}(\mathrm{x}, \mathrm{y}) \subseteq \mathrm{T}_{\mathrm{G}_{\mathrm{k}}}(\mathrm{x}, \mathrm{y})$ for all $(\mathrm{x}, \mathrm{y}) \varepsilon \mathrm{N}$ and for all k. If $\Phi\left(\mathrm{Q} ; \mathrm{x}_{1}, \mathrm{x}\right)$ is the generalized polar of the product $\mathrm{Q}(\mathrm{x})(\mathrm{cf} .(2.1)-(2.3))$ with $\mathrm{m}_{\mathrm{k}}>$ o for $\mathrm{k} \leq \mathrm{p}(<\mathrm{q})$ and $\mathrm{m}_{\mathrm{k}}<$ o for $\mathrm{k}>\mathrm{p}$, then $\mathrm{Q}\left(\mathrm{Q} ; \mathrm{x}_{1}, \mathrm{x}\right) \neq 0$ for all Zinearly independent elements $\mathbf{x}, \mathrm{x}_{1}$ of $\mathrm{E}$ such that $\mathrm{x}_{1} \varepsilon \mathrm{E}-\underset{\mathrm{k}=1}{\mathrm{u}} \mathrm{E}_{\mathrm{o}}^{(\mathrm{k})}$ and $\mathrm{x} \varepsilon \mathrm{E}-\left(\underset{\mathrm{k}=1}{\mathrm{q}} \mathrm{E}_{\mathrm{o}}^{(\mathrm{k})}\right) \cup \mathrm{T}_{\mathrm{S}}\left(\mathrm{x}_{\mathrm{o}}, \mathrm{y}_{\mathrm{o}}\right)$, where $\left(\mathrm{x}_{\mathrm{o}}, \mathrm{y}_{\mathrm{o}}\right)$ is the unique element in $\mathrm{N} \cap \mathcal{L}\left[\mathrm{x}, \mathrm{x}_{1}\right], \mathrm{x}_{1}=\gamma \mathrm{x}_{0}+\delta \mathrm{y}_{\mathrm{o}}$ and 


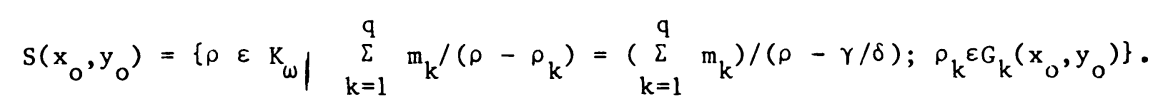

REMARK. Let us note that $\rho=\omega$ must belong to $S\left(x_{0}, y_{0}\right)$ in the case when $\gamma / \delta \neq \omega$

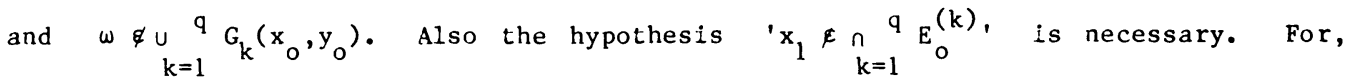
otherwise, $S\left(x_{0}, y_{0}\right)$ would be all of $k$ and the theorem would become uninteresting.

PROOF. Let $x, x_{1}$ be linearly independent elemenis of $E$ such that $x \notin\left(u_{k=1}^{q} E_{o}^{(k)}\right) \cup T_{S}\left(x_{0}, y_{0}\right)$ and $x_{1} \notin \operatorname{n}_{k=1}^{q} E_{o}^{(k)}$, where $\left(x_{0}, y_{0}\right)$ is the unique element in $N \cap \mathcal{C}^{2}\left[\mathrm{x}, \mathrm{x}_{1}\right]$ (cf. definition of nucleus [5]). Then there exists a unique set of scalars $\alpha, \beta, \gamma, \delta$ (with $\alpha \delta-\beta \gamma \neq 0$ ) such that $x_{1}=\alpha x_{0}+\beta y_{0}$ and $x_{1}=\gamma x_{0}+\delta y_{0}$. obviously, the choice of $x$ implies that $\alpha / \beta \notin\left(u_{k=1}^{q} G_{k}\left(x_{0}, y_{0}\right)\right) u s\left(x_{0}, y_{0}\right)$, due to the notation in (2.5). We claim that $\alpha / \beta \neq \omega$. This is trivial when $\gamma / \delta=\omega$ (since $\delta=0$ and $\alpha \delta-\beta \gamma \neq 0)$. It is obvious also when $\gamma / \delta \neq \omega$ and $\omega$ belongs to $u_{k=1}^{q} G_{k}\left(x_{0}, y_{0}\right)$. However, in case $\gamma / \delta \neq \omega$ and $\omega \notin u_{k=1}^{q} G_{k}\left(x_{0}, y_{0}\right)$, the definition of $S\left(x_{0}, y_{0}\right)$ says that $\omega$ must belong to $S\left(x_{0}, y_{0}\right)$. So that $\alpha / \beta \neq \omega$ in all cases.

The fact that $K$ is algebraically closed allows us to write, for each $k=1,2, \ldots, q$,

$$
P_{k}\left(s x+t x_{1}\right)=\prod_{j=1}^{n_{k}}\left(\delta_{j k} s-\gamma_{j k} t\right) \text {. }
$$

Since $P_{k}(x)=\prod_{j=1}^{n_{k}} \delta_{j k} \neq 0$ for all $k$, we have that for each $k(1 \leq k \leq q) \delta_{j k} \neq 0$ for all $j=1,2, \ldots, n_{k}$. If we set $\rho_{j k}=\gamma_{j k} / \delta_{j k}$ then, using the same technique as in the beginning of the proof of Theorem 2.5 due to Zaheer [5], we conclude that

$$
\rho_{j k} \varepsilon U\left(G_{k}\left(x_{o}, y_{o}\right)\right) \text { for } j=1,2, \ldots, n_{k}, \quad 1 \leq k \leq q
$$

and, further, that $U\left(G_{k}\left(x_{0}, y_{o}\right)\right)$ are $K_{0}$-convex $g \cdot c \cdot r \cdot s$ of $K$, where $U$ is the homographic transformation [21] of $K_{\omega}$ given by $U(\rho)=(\delta \rho-\gamma) /(-\beta \rho+\alpha)$. Therefore, (3.1) and the $K_{0}$-convexity of $U\left(G_{k}\left(x_{0}, y_{0}\right)\right)$ give

$$
\mu_{k}(s \text { ay })=\sum_{j=1}^{n_{k}}\left(1 / n_{k}\right) \rho_{j k} \varepsilon U\left(G_{k}\left(x_{o}, y_{o}\right)\right) \text { for } k=1,2, \ldots, q \text {. }
$$

This implies that there exist elements $\rho_{k} \varepsilon G_{k}\left(x_{0}, y_{0}\right)$ such that

$$
\mu_{k}=U\left(\rho_{k}\right)=\left(\delta \rho_{k}-\gamma\right) /\left(-\beta \rho_{k}+\alpha\right) \neq \omega \text { for } k=1,2, \ldots, q \text {. }
$$

Let us write

$$
\nu_{k}=m_{k} \mu_{k}=\sum_{j=1}^{n_{k}}\left(m_{k} / n_{k}\right) \rho_{j k} \quad \forall k=1,2, \ldots, q .
$$

We now claim that $v_{1}+v_{2}+\ldots+v_{q} \neq 0$. First we notice that the $\nu_{k}$ 's cannot vanish simultaneously. For, otherwise, $\gamma / \delta$ would belong to all the g.c.r.'s $G_{k}\left(x_{0}, y_{0}\right)$ 
for $k=1,2, \ldots, q$, which in turn would imply that

$$
x_{1}=r x_{0}+\delta y_{0} \varepsilon \underset{k=1}{n} T_{G_{k}}\left(x_{0}, y_{0}\right) \subseteq \underset{k=1}{n} E_{0}^{(k)}
$$

This contradicts the fact that $x_{1} \notin{ }_{k=1}^{n} \mathrm{E}_{0}^{(k)}$. Therefore, in order to establish the said claim, it remains only to deal with the case when at least any two of the $\nu_{k}$ 's do not vanish (since the claim is obvious otherwise). Now, with this assumption, suppose on the contrary that $\nu_{1}+\nu_{2}+\ldots+\nu_{q}=0$. Then equations (3.3) amd (3.4) would imply that

$$
\sum_{k=1}^{q} m_{k}\left(\delta \rho_{k}-\gamma\right) /\left(-\beta \rho_{k}+\alpha\right)=0 \text {. }
$$

Since $\alpha / \beta \neq \omega$, we see that $\beta \neq 0$ and, consequently, the last equation can be written as

Therefore,

$$
\sum_{k=1}^{q} m_{k}\left[-\delta / \beta+\{(\alpha \delta / \beta)-\gamma\} /\left(-\beta \rho_{k}+\alpha\right)\right]=0
$$

$$
\begin{gathered}
\quad(\Delta / \beta) \sum_{k=1}^{q} m_{k} /\left(-\beta \rho_{k}+\alpha\right)=(\delta / \beta) \underset{k=1}{\sum_{k}^{q} m_{k},} \\
\text { where } \Delta=\alpha \delta-\beta \gamma \neq 0.0 r, \\
\sum_{k=1}^{q} m_{k} /\left(\alpha / \beta-\rho_{k}\right)=\frac{\delta \beta}{\alpha \delta-\beta \gamma}\left(\sum_{k=1}^{q} m_{k}\right)=\frac{\delta}{\delta(\alpha / \beta)-\gamma}\left(\sum_{k=1}^{q} m_{k}\right) .
\end{gathered}
$$

That is, irrespective of whether $\delta=0$ or $\delta \neq 0$, we get

$$
\sum_{k=1}^{q} m_{k} /\left(\alpha / \beta-\rho_{k}\right)=\left(\sum_{k=1}^{q} m_{k}\right) /(\alpha / \beta-\gamma / \delta),
$$

where $\rho_{k} \in G_{k}\left(x_{0}, y_{0}\right)$ for $k=1,2, \ldots, q,\left(x_{0} y_{0}\right) \varepsilon N \cap L^{2}\left[x, x_{1}\right]$ and $x_{1}=\gamma x_{0}+\delta y_{0}$. This implies that $\alpha / \beta \varepsilon s\left(x_{0}, y_{0}\right)$ and, hence, that $x=\alpha x_{0}+\beta y_{0} \varepsilon T_{S}\left(x_{0}, y_{0}\right)$, contradicting the choice of $x$ already made. Therefore $v_{1}+v_{2}+\ldots+v_{q} \neq 0$. But we know ([5] or [3]) that

$$
\begin{aligned}
P\left(Q ; x_{1}, x\right) & =-\left[\sum_{k=1}^{q} \sum_{j=1}^{\eta_{k}}\left(m_{k} / n_{k}\right) \rho_{j k}\right] \cdot \prod_{k=1}^{q} P_{k}(x) \\
& \left.=-\left(\sum_{k=1}^{q} \nu_{k}\right) \cdot \prod_{k=1}^{q} P_{k}(x) \quad \text { (due to }(3.4)\right) .
\end{aligned}
$$

Since $P_{k}(x) \neq 0$ for all $k$ and since $\nu_{1}+\ldots+\nu_{q} \neq 0$, the proof is complete. If we take $\sum_{k=1}^{q} m_{k}=0$ in the above theorem, the set $s\left(x_{0}, y_{0}\right)$ remains unchanged when $x_{1}$ varies freely in $L\left[x_{0}, y_{0}\right]$ subject to the condition that $x_{1} \&{\underset{k=1}{n}}_{E_{0}}^{(k)}$. 
In order to get a simpler and more interesting version in which $x_{1}$ varies freely over all of $E$ it is desirable to further assume that $\underset{k=1}{n}{ }_{0}^{(k)}=\phi$. We do precisely this to obtain the following theorem which deals exclusively with generalized polars having a vanishing weight.

THEOREM 3.2. Under the notations and hypotheses of Theorem 3.1 if we assume that $\underset{k=1}{n} E_{0}^{(k)}=\phi$ and $\sum_{k=1}^{q} m_{k}=0$, then $P\left(Q ; x_{1}, x\right) \neq 0$ for all linearly independent elements $\mathrm{x}, \mathrm{x}_{1}$ of $\mathrm{E}$ such that $\mathrm{x} \in \mathrm{E}-\mathrm{u}_{\mathrm{k}=1}^{\mathrm{q}+1} \mathrm{E}_{\mathrm{o}}^{(\mathrm{k})}$, where $\mathrm{E}_{\mathrm{o}}^{(\mathrm{q}+1)}=\mathrm{E}_{\mathrm{o}}\left(\mathrm{N}, \mathrm{G}_{\mathrm{q}+1}\right)$ is the cone defined by

$$
G_{q+1}\left(x_{o}, y_{o}\right)=\left\{\begin{array}{lll}
\rho & \varepsilon & K_{\omega}
\end{array} \sum_{k=1}^{q} m_{k} /\left(\rho-\rho_{k}\right)=0 ; \rho_{k} \varepsilon G_{k}\left(x_{0}, y_{o}\right)\right\}
$$

for alz $\left(\mathrm{x}_{\mathrm{o}}, \mathrm{y}_{\mathrm{o}}\right) \varepsilon \mathrm{N}$.

PROOF. If $x, x_{1}$ are any linearly independent elements such that $x_{1} \varepsilon E$ and $x \notin u_{k=1}^{q+1} E_{0}^{(k)}$, then there exists a unique element $\left(x_{0} y_{0}\right) \varepsilon N \cap L^{2}\left[x, x_{1}\right]$ such that $x_{1}=\gamma x_{0}+\delta y_{0}$ and, in the present set up, $s\left(x_{0}, y_{0}\right)=G_{q+1}\left(x_{0}, y_{0}\right) \quad\left(\right.$ since $\left.\sum_{k=1}^{q} m_{k}=0\right)$. That is, $x_{1} \notin \underset{k=1}{q} \mathrm{E}_{0}^{(k)}=\phi$ and $x \notin\left(\underset{k=1}{\left(u_{0}\right.} E_{0}^{(k)}\right) \cup T_{S}\left(x_{0}, y_{0}\right)$. Now the proof follows from Theorem 3.1 .

As application of Theorem 3.1 in the complex plane we prove the following corollary which, apart from generalizing the two-circle theorem and the cross-ratio theorem of Walsh [22] (cf. also [7], Theorems 20,1 and 22,2), improves upon Marden's general theorem on critical points of rational functions ([7] or [23] and [24]). In the following, $Z(f)$ denotes the set of all zeros of $f$.

COROLLARY 3.3. For each $\mathbf{k}=0,1, \ldots, \mathrm{p}$, let $\mathrm{f}_{\mathbf{k}}(\mathrm{z})$ be a polynomial (from $\mathbf{C}$ to $\mathbf{C})$ of degree $\mathrm{n}_{\mathrm{k}}$. If $\mathrm{c}_{\mathrm{k}} \in \mathrm{D}\left(\mathbf{c}_{\omega}\right)$ such that $\mathrm{Z}\left(\mathrm{f}_{\mathrm{k}}\right) \subseteq \mathrm{C}_{\mathrm{k}}$ for $\mathrm{k}=0,1, \ldots, \mathrm{p}$ and if $\omega \notin{ }_{k=0}^{P} C_{k}$, then every finite zero of the derivative of the rational function

$$
f(z)=\frac{f_{o}(z) f_{1}(z) \ldots f_{q}(z)}{f_{q+1}(z) f_{q+2}(z) \ldots f_{p}(z)} \quad(q<p)
$$

Zies in $\mathrm{u}_{\mathbf{k}=0}^{\mathrm{P}+1} \mathrm{C}_{\mathbf{k}}$, where

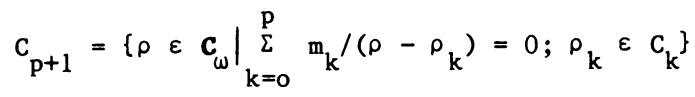

and $\mathrm{m}_{\mathrm{k}}=\mathrm{n}_{\mathrm{k}}$ or $-\mathrm{n}_{\mathrm{k}}$ according as $\mathrm{k} \leq \mathrm{q}$ or $\mathrm{k}>\mathrm{q}$.

PROOF. In view of Remark 2.2 (II), the sets

$$
E_{0}^{(k)} \equiv E_{o}\left(N, G_{k}\right)=\left\{s x_{0}+t y_{0} \neq 0 \mid s, t \varepsilon C ; s / t \varepsilon C_{k}\right\}, 0 \leq k \leq p,
$$

where $x_{0}=(1,0), y_{0}=(0,1), N=\left\{\left(x_{0}, y_{0}\right)\right\}$ and $G_{k}\left(x_{0}, y_{0}\right)=C_{k}$, are circular cones in $c^{2}$ such that $x_{0}=(1,0) \varepsilon \prod_{k=0}^{p} E_{o}^{(k)}$. Letting $f_{k}(z)=\sum_{j=0}^{n^{k}} a_{j k} z^{j}, 0 \leq k \leq p$, we 
define the mappings $P_{k}: C^{2} \rightarrow C$ by

$$
P_{k}(x) \equiv P_{k}\left(s x_{0}+t y_{0}\right)=\sum_{j=0}^{n k} a_{j k} s^{j} t^{n_{k}-j} \quad \forall x=(s, t) \varepsilon c^{2} .
$$

Then $P_{k}$ is an a.h.p. of degree $n_{k}$ from $c^{2}$ to $c$ such that $\mathrm{Z}_{\mathrm{P}_{k}}\left(\mathrm{x}_{0}, \mathrm{y}_{0}\right) \subseteq \mathrm{T}_{G_{k}}\left(\mathrm{x}_{0}, \mathrm{y}_{0}\right)$ for $\mathrm{k}=0,1, \ldots, \mathrm{p}$. This is so because

$$
P_{k}(x) \equiv P_{k}\left(s x_{0}+t y_{0}\right)=t^{n_{k}} f_{k}(s / t) \quad \forall x=(s, t) \neq 0
$$

and because $\mathrm{Z}\left(\mathrm{f}_{\mathrm{k}}\right) \subseteq \mathrm{C}_{\mathrm{k}}=\mathrm{T}_{G_{\mathrm{k}}}\left(\mathrm{x}_{\mathrm{o}}, \mathrm{y}_{\mathrm{o}}\right)$. Now we consider the generalized polar $\mathrm{Q}\left(\mathrm{Q} ; \mathrm{x}_{1}, \mathrm{x}\right)$ of the product $Q(x)$ of these a.h.p.'s, with $m_{k}=n_{k}$ or $-n_{k}$ according as $k \leq q$ or $k>q$. If we take $x_{1}=x_{0}=(1,0)$ (so that $s_{1}=1$ and $t_{1}=0$ ), we see as in [5], that

$$
P_{k}\left(x_{0}, x\right)=\left(1 / n_{k}\right) \partial P_{k} / \partial s=\left(1 / n_{k}\right) t^{n_{k}-1} f_{k}^{\prime}(s / t)
$$

for $k=0,1, \ldots, p$. If we set $n_{0}+n_{1}+\ldots+n_{p}-1=m$ and define

$$
F_{k}(z)=f_{0}(z) f_{1}(z) \ldots f_{k-1}(z) \cdot f_{k}^{\prime}(z) \cdot f_{k+1}(z) \ldots f_{p}(z)
$$

equations (3.7) and (3.8) imply that, for $x=(s, t) \varepsilon c^{2}$,

$$
\begin{aligned}
P\left(Q ; x_{0}, x\right) & =t^{m} \cdot \sum_{k=0}^{p}\left(m_{k} / n_{k}\right) F_{k}(s / t) \\
& =t^{m}\left[\sum_{k=0}^{q} F_{k}(s / t)-\sum_{k=q+1}^{p} F_{k}(s / t)\right] \\
& =t^{m} \cdot f^{\prime}(s / t) \cdot\left[f_{q+1}(s / t) \ldots f_{p}(s / t)\right]^{2} .
\end{aligned}
$$

Since $\mathrm{x}_{1}=\mathrm{x}_{0} \notin \underset{\mathrm{k}=0}{\mathrm{P}} \mathrm{E}_{0}^{(\mathrm{k})}$ and since Theorem 3.1 is applicable in the present set up (with $\gamma=1$ and $\delta=0$, so that $\gamma / \delta=\omega)$, it implies that $\Phi\left(Q ; x_{1}, x\right) \neq 0$ whenever the element $x=(s, t)$ is linearly independent to $x_{0}$ such that $x \notin u_{k=0}^{p+1} E_{0}^{(k)}$, where

$$
E_{0}^{(p+1)} \equiv E_{o}\left(N, G_{p+1}\right)=\left\{s x_{0}+t y_{0} \neq 0 \mid s, t \varepsilon c ; s / t \varepsilon G_{p+1}\left(x_{o}, y_{o}\right)\right\}
$$

and where

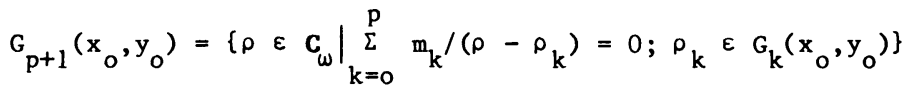

$$
\begin{aligned}
& =\left\{\begin{array}{llll}
\rho & \varepsilon & \mathbf{C}_{\omega} & \sum_{k=0}^{p} m_{k} /\left(\rho-\rho_{k}\right)=0 ; \rho_{k} \varepsilon C_{k}
\end{array}\right\} \\
& =\mathrm{C}_{\mathrm{p}+1} \text {, (due to the choice of } \mathrm{m}_{\mathrm{k}} \text { made above). }
\end{aligned}
$$

That is, $\Phi\left(Q ; x_{0}, x\right) \neq 0$ for all elements $x=(s, t)$ for which $t \neq 0$ and for which 


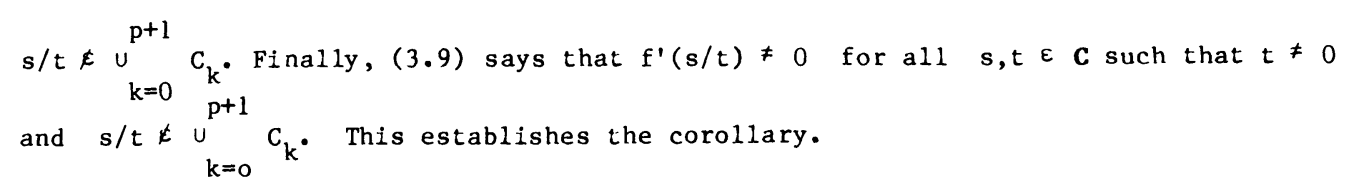

REMARK 3.4. (I) In the special case when the g.c.r.'s $c_{k}$ are specialized as the closed interior or the closed exterior of circles, we claim that the above corollary reduces essentially to Theorem 21,1 of Marden [7]. This is upheld by the following arguments: If the $C_{k}$ are taken to be the regions $\sigma_{k} C_{k}(z) \leq 0$ of Marden's Theorem 21,1, then Lemma 21,1 of Marden [7] and the succeeding arguments therein show that the region $\bigcup_{k=0}^{p+1} C_{k}$ in our corollary is precisely the region satisfying the p+2 inequalities 21,3 in Marden's theorem.

(II) In what follows we show that Corollary 3.3 holds as such when $C$ is replaced by $K$, provided the term 'derivative' is replaced by 'formal derivative'. We know by ([5] or [12]) that the polynomial $f^{\prime}(z)=\sum_{k=1}^{n} k a_{k} z^{k-1}$ is called the formal derivative of the polynomial $f(z)=\sum_{k=0}^{n} a_{k} z^{k}$ from $k$ to $k$ and that

$$
\left(f_{1} f_{2} \ldots f_{n}\right)^{\prime}=\sum_{k=1}^{n} f_{1} f_{2} \ldots f_{k-1} f_{k}^{\prime} f_{k+1} \ldots f_{n} \text {, }
$$

where the $f_{i}$ are polynomials [12]. If we now define the formal derivative of the quotient $f_{1} / f_{2}$ ( $f_{i}$ being polynomials) to be given by $\left(f_{1} / f_{2}\right)^{\prime}=\left(f_{1}^{\prime} f_{2}-f_{1} f_{2}^{\prime}\right) /\left(f_{2}\right)^{2}$, then the formal derivative of the quotient

$$
f_{0}(z) f_{1}(z) \ldots f_{q}(z) / f_{q+1}(z) \ldots f_{p}(z)
$$

is given by equation (3.9).

$$
\left[\sum_{k=0}^{q} F_{k}(z)-\sum_{k=q+1}^{p} F_{k}(z)\right] /\left[f_{q+1}(z) \ldots f_{p}(z)\right]^{2} .
$$

In view of the definition of the formal derivative $f^{\prime}(z)$ of a polynomial $f(z)$ from $K$ to $K$ and of formal partial derivatives $\partial \mathrm{P} / \partial \mathrm{s}$ of a polynomial $\mathrm{P}(\mathrm{s}, t) \mathrm{from}$ $\mathrm{K}^{2}$ to $\mathrm{K}$ [5], we can easily show that Corolzary 3.3 stizl holds when $\mathbf{C}$ is replaced by K. The proof proceeds exactly on the lines of the proof of corollary 3.3, except only that we replace $\mathrm{C}$ by $\mathrm{K}$ all along. Let us point out that the expression (3.10) is precisely the formal derivative of the function $f(z)$ in (3.5) and it justifies the validity of steps (3.8) and (3.9) in the proof of Corollary 3.3.

(III) We remark that in Corollary 3.3, we must add the hypothesis ' $n_{k=0}^{p} C_{k}=\Phi^{\prime}$, in order to have a nontrivial result for the rational function $f(z)$ with

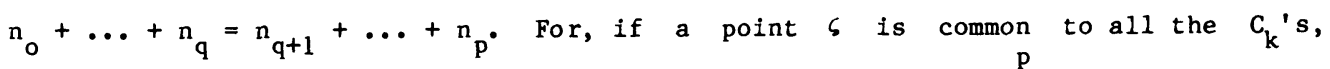
then fixing $\rho_{0}=\rho_{1}=\ldots \rho_{p}=\xi$ in (3.6) we see that (since $\underset{k=0}{\stackrel{p}{k} m_{k}}=0$ ) $\sum_{k=0}^{p} m_{k} /\left(\rho-\rho_{k}\right)=\left(\sum_{k=0}^{p} m_{k}\right) /(\rho-\zeta)=0 \quad \forall \rho \in \mathbf{C}$

and, hence, that $\mathrm{c}_{\mathrm{p}+1}=\mathbf{C}$. 
(IV) It has been shown by Marden [7] that Walsh's cross-ratio Theorem 22,2, is a special case of Marden's general Theorem 21,1, but on 1y in terms of closed interior or closed exterior of circles (a proper subclass of $D\left(\mathbf{C}_{\omega}\right)$ ). Whereas, our Corollary 3.3 validates Walsh's theorem in terms of g.c.r.'s. In fact, applying our corollary in the set up of Walsh's theorem with $C_{i}$ 's taken as g.c.r.'s, we conclude that every finite zero of the derivative of the function $f_{1}(z) f_{2}(z) / f_{3}(z)$ lies in $u_{i=1}^{4} C_{i}$, where

$$
C_{4}=\left\{\rho \varepsilon C_{\omega} \mid n_{1}\left(\rho-\rho_{1}\right)+n_{2} /\left(\rho-\rho_{2}\right)-n_{3} /\left(\rho-\rho_{3}\right)=0 ; \rho_{i} \varepsilon C_{i}\right\}
$$

In veiw of Lemma 4.2 of the next section, we see that $C_{4}=H_{\lambda}$ for $K=C, \lambda=n_{2} / n_{1}$ $G_{i}=C_{i}$, and that

$$
\left(C_{4}-\{\omega\}\right)-u_{i=1}^{3} C_{i}=\left(R_{\lambda}-\{\omega\}\right)-u_{i=1}^{3} C_{i},
$$

where

$$
R_{\lambda}=\left\{\rho \in C_{\omega} \mid\left(\rho, \rho_{3}, \rho_{2}, \rho_{1}\right)=-n_{2} / n_{1} ; \rho_{i} \varepsilon C_{i}\right\}
$$

Consequently, every finite zero of the derivative of the said function lies in $C_{1} \cup C_{2} u$ $\mathrm{C}_{3} \cup \mathrm{C}$, where $\mathrm{C}=\mathrm{R}_{\lambda}-\{\omega\}$. This shows that an improved version of Walsh's cross-ratio theorem follows from Corollary 3.3 .

(V) It may be observed that an improved form of Walsh's two-circle theorems in its complete form ([5], Corollaries 2.8 and 4.3) may also be obtained from the above corollary. To this effect we apply Corollary 3.3 in the set up of Zaheer's Corollary 4.3 [5], with the $D_{i}^{\prime}$ 's replaced by g.c.r.'s $C_{i}$ such that $\omega \varepsilon C_{1} \cap C_{2}$, and conclude that every finite zero of the derivative of the function $f_{1}(z) / f_{2}(z) \quad 1$ ies in $u_{i=1}^{3} C_{i}$, where

$$
\begin{aligned}
& C_{3}\left\{\begin{array}{lll}
\rho & \varepsilon & C_{\omega} \mid n_{1} /\left(\rho-\rho_{1}\right)-n_{2} /\left(\rho-\rho_{2}\right)=0 ; \rho_{i} \varepsilon C_{i}
\end{array}\right\} \\
&=\left\{\begin{array}{lll}
\rho & \varepsilon & C_{\omega} \mid \rho=\left(n_{1} \rho_{2}-n_{2} \rho_{1}\right) /\left(n_{2}-n_{i}\right) ; \rho_{i} \varepsilon C_{i}
\end{array}\right\} .
\end{aligned}
$$

We point out that, in case the $C_{i}^{\prime}$ s are taken as the regions $D_{i}$ of Corollary 4.3 of Zaheer [5], the region $C_{3}$ is precisely $D\left(c_{3}, r_{3}\right)$ (cf. notation there) and we are done. In the case when $n_{1}=n_{2}$ and $c_{i} \cap c_{2}=\phi$, the conclusion just drawn still holds, but in this case the region $C_{3}$ is empty, and we are done with corollary 2.8 in [5].

4. THE CASE OF ALGEB RA-VALUEd generalized POLARS.

Our aim in this section is to obtain a more general formulation of Theorem 3.1 that could answer the corresponding problem for algebra valued generalized polars having an arbitrary weight. In fact, it will be shown that, whereas the main theorem of this section does include in it the main theorem of the preceding section, it also incorporates into it a variety of other known results. First, we describe some concepts and establish some results that we need in this section. We refer [17], [1] and [2] for the following material. 
A subset of $M$ of $V$ is called fully supportable (initially termed as 'A-supportable' by Zaheer [9]) if every point $\xi$ outside $M$ is contained in some ideal maximal subspace of $V$ which does not meet $M$. In other words, for every $\xi \varepsilon V-M$, there is a unique nontrivial scalar homomorphism $L$ on $V$ such that $L(\xi)=0$ but $L(v) \neq 0$ for every $v \in M[18]$. If $M$ is a fully supportable subset of $v$, then $M$ is a supportable subset of $V$ (regarded as a vector space), but not conversely (for definition of supportable subsets see [6]). We remark that the complement in $V$ of every ideal maximal subspace of $V$ is a fully supportable subset of $V$. Given $P \varepsilon P_{n}^{*}$ and a fully supportable subset $M$ of $V$, we shall write, for given $x, y \in E$,

$$
E_{P}(x, y)=\{s x+t y \neq 0 \mid s, t \varepsilon K ; P(s x+t y) \notin M\}
$$

REMARK 4.1. Since identity map from $K$ to $K$ is the only nontrivial scalar homomorphism on $K$, the set $M=K-\{$ o $\}$ is the only fully supportable subset of $K$ ( $t$ ake $V=K$ in the definition) and the corresponding set $E_{P}(x, y)$, as given by (4.1), becomes the null-set $\mathrm{z}_{\mathrm{P}}(\mathrm{x}, \mathrm{y})$ of $\mathrm{P}$ as defined in the beginning of Section 3 .

In the next few lemmas, the notation $\left(\rho_{,} \rho_{1}, \rho_{2}, \rho_{3}\right)$ stands for the crossratio of an element $\rho \varepsilon \mathrm{K}_{\omega}$ with respect to given distinct elements $\rho_{1}, \rho_{2}, \rho_{3} \varepsilon \mathrm{K}_{\omega}$ and it designates a unique element in $\mathrm{K}_{\omega}$ (for definition and other relevant details see [5]).

LEMMA 4.2. Given an element $\lambda>0$ in $\mathrm{K}$ and g.c.r.'s $\mathrm{G}_{i} \in \mathrm{D}\left(\mathrm{K}_{\omega}\right)$ for $i=1,2,3$, let us define

$$
H_{\lambda}=\left\{\begin{array}{lll}
\rho & \varepsilon & K_{\omega}
\end{array} \mid 1 /\left(\rho-\rho_{1}\right)+\lambda /\left(\rho-\rho_{2}\right)-(1+\lambda) /\left(\rho-\rho_{3}\right)=0 ; \rho_{i} \varepsilon G_{i}\right\}
$$

and

$$
\mathrm{R}_{\lambda}=\left\{\begin{array}{lll}
\rho & \varepsilon \mathrm{K}_{\omega}
\end{array} \mid\left(\rho, \rho_{3}, \rho_{2}, \rho_{1}\right)=-\lambda ; \rho_{i} \varepsilon \mathrm{G}_{i}\right\} \text {. }
$$

If $G_{1} \cap G_{2} \cap G_{3}=\phi$, then

$$
\left(H_{\lambda}-\{\omega\}\right)-u_{i=1}^{3} G_{i}=\left(R_{\lambda}-\{\omega\}\right)-u_{i=1}^{3} G_{i} \cdot
$$

PROOF. In order to prove the lemma it is sufficient to show that, if $\rho \notin G_{1} \cup G_{2} \cup G_{3} \cup\{\omega\}$ and $\rho_{1} \in G_{i}(i=1,2,3)$, the equation

$$
1 /\left(\rho-\rho_{1}\right)+\lambda /\left(\rho-\rho_{2}\right)-(1+\lambda) /\left(\rho-\rho_{3}\right)=0
$$

holds true if and only if the equation

$$
\left(\rho, \rho_{3}, \rho_{2}, \rho_{1}\right)=-\lambda
$$

holds true. First, we claim that none of these equations can hold unless $\rho_{1}, \rho_{2}, \rho_{3}$ are distinct elements of $\mathrm{K}_{\omega}$. This is obvious in case of (4.3) due to the definition of cross-ratio. In case of (4.2), this follows from the fact that if any two of the $\rho_{i}$ 's coincide and if (4.2) holds then all the three must coincide, contradicting that $G_{1} \cap G_{2} \cap G_{3}=\phi$. Therefore, we assume that $\rho_{1}, \rho_{2}, \rho_{3}$ are distinct elements of $K_{\omega}$ and so we divide the proof into the following two cases: 
Case (i). $\rho_{1}, \rho_{2}, \rho_{3} \neq \omega$. In this case, since $\rho, \rho_{1}, \rho_{2}, \rho_{3}$ are distinct elements of $K$, the equation (4.2) holds if and only if

$$
\left(\rho_{1}-\rho_{3}\right) /\left(\rho-\rho_{1}\right)\left(\rho-\rho_{3}\right)+\lambda\left(\rho_{2}-\rho_{3}\right) /\left(\rho-\rho_{2}\right)\left(\rho-\rho_{3}\right)=0
$$

or, if and on $1 y$ if

$$
\left(\rho-\rho_{2}\right)\left(\rho_{3}-\rho_{1}\right) /\left(\rho-\rho_{1}\right)\left(\rho_{3}-\rho_{2}\right)=-\lambda \text {. }
$$

This is true if and only if $\left(\rho, \rho_{3}, \rho_{2}, \rho_{1}\right)=-\lambda$. That is, (4.2) holds if and only if (4.3) holds.

Case (ii). One of the $\rho_{i}^{\prime} s$ is $w$. In this case, let us point out that $\rho, \rho_{1}, \rho_{2}, \rho_{3}$ are distinct elements of $K_{\omega}$ with only one of the $\rho_{i}$ 's being $\omega$. Therefore, the equation $(4.2)$ is equivalent to the equation

$$
\begin{aligned}
& \lambda /\left(\rho-\rho_{2}\right)=(1+\lambda) /\left(\rho-\rho_{3}\right), \\
& 1 /\left(\rho-\rho_{1}\right)=(1+\lambda) /\left(\rho-\rho_{3}\right),
\end{aligned}
$$

ot

$$
1 /\left(\rho-\rho_{1}\right)=-\lambda /\left(\rho-\rho_{2}\right)
$$

according as $\rho_{1}=\omega, \rho_{2}=\omega$ or $\rho_{3}=\omega$, respectively. Now, the equations (4.4) (4.6) are, respectively, equivalent to the equations $\left(\rho-\rho_{2}\right) /\left(\rho_{3}-\rho_{2}\right)=-\lambda$, $\left(\rho_{3}-\rho_{1}\right) /\left(\rho-\rho_{1}\right)=-\lambda$, and $\left(\rho-\rho_{2}\right) /\left(\rho-\rho_{1}\right)=-\lambda$, in the respective cases under consideration. The definition of cross-ratio implies that each of the equations (4.2) holds if and only if $\left(\rho, \rho_{3}, \rho_{2}, \rho_{1}\right)=-\lambda$. That is (4.2) holds if and only if (4.3) holds.

Cases (i) and (ii) complete our proof.

LEMMA 4.3. Let $\mathrm{G}_{i} \varepsilon \mathrm{D}\left(\mathrm{K}_{\omega}\right)$ for $\mathrm{i}=1,2,3$, and let $\mathrm{m}_{\mathbf{k}} \varepsilon \mathrm{K}-\{0\}$ for $\mathbf{k}=$ $1,2, \ldots, \mathrm{q}$ such that $\mathrm{m}_{\mathrm{k}}>0$ for $\mathrm{k} \leq \mathrm{p}(\mathrm{P}<\mathrm{q})$ and $\mathrm{m}_{\mathrm{k}}<0$ for $\mathrm{k}>\mathrm{p}$. Giver. any $\mathrm{r}, 1 \leq \mathrm{r}<\mathrm{p}$, and $a \quad \zeta \in \mathrm{k}_{\omega}$, we define

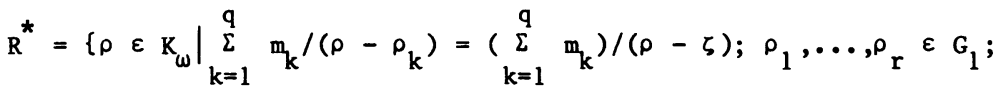

$$
\begin{aligned}
& \left.\rho_{\mathrm{r}+1}, \ldots, \rho_{\mathrm{p}} \in \mathrm{G}_{2} ; \rho_{\mathrm{p}+1}, \ldots, \rho_{\mathrm{q}} \varepsilon \mathrm{G}_{3}\right\} \text {, }
\end{aligned}
$$

and

$$
H^{*}=\left\{\begin{array}{lll}
\rho & \varepsilon & K_{\omega}
\end{array} \sum_{i=1}^{3} A_{i} /\left(\rho-\rho_{1}\right)=\left(\sum_{i=1}^{3} A_{i}\right) /(\rho-\zeta) ; \rho_{1} \varepsilon G_{i}\right\},
$$

with $A_{1}=\sum_{k=1}^{r} m_{k}, A_{2}=\sum_{k=r+1}^{p} m_{k}, A_{3}=\sum_{k=p+1}^{q} m_{k} \cdot$ with $\zeta \& \underbrace{3}_{i=1} G_{i}$,

then

$$
\left(R^{*}-\{\zeta\}\right)-u_{i=1}^{3} G_{.}=\left(H^{*}-\{\zeta\}\right)-u_{i=1}^{3} G_{i}
$$

PROOF. If $\rho \varepsilon\left(H^{*}-\stackrel{i=1}{\{\zeta\}}\right)-U_{i=1}^{3} G_{i}$, then there exist elements $\rho_{i} \varepsilon G_{i}$ $(i=1,2,3)$ such that $\rho \in G_{1} \cup \boldsymbol{G}_{2} \cup G_{3} \cup\{\zeta\}$ and

$$
A_{1} /\left(\rho-\rho_{1}\right)+A_{2} /\left(\rho-\rho_{2}\right)+A_{3} /\left(\rho-\rho_{3}\right)=\left(A_{1}+A_{2}+A_{3}\right) /(\rho-\zeta) \text {. }
$$


Obviously, $\rho \neq \rho_{1}, \rho_{2}, \rho_{3}, \zeta$. If we choose elements $\rho_{k}^{\prime}, k=1,2, \ldots, q$, such that

$$
\rho_{k}^{\prime}=\left\{\begin{array}{l}
\rho_{1} \text { for } k=1,2, \ldots, r \\
\rho_{2} \text { for } k=r+1, \ldots, p \\
\rho_{3} \text { for } k=p+1, \ldots, q,
\end{array}\right.
$$

then equation $(4.7)$ can be written as

$$
\sum_{k=1}^{r} m_{k} /\left(\rho-\rho_{k}^{\prime}\right)+\sum_{k=r+1}^{p} m_{k} /\left(\rho-\rho^{\prime}{ }_{k}\right)+\sum_{k=p+1}^{q} m_{k} /\left(\rho-\rho_{k}^{\prime}\right)=\left(\sum_{k=1}^{q} m_{k}\right) /(\rho-\zeta)
$$

or

$$
\sum_{k=1}^{q} m_{k} /\left(\rho-\rho_{k}^{\prime}\right)=\left(\sum_{k=1}^{q} m_{k}\right) /(\rho-\zeta)
$$

This implies that $\rho \varepsilon\left(R^{*}-\{\zeta\}\right)-\bigcup_{i=1}^{3} G_{i} \cdot$ Hence

$$
\left(H^{*}-\{\zeta\}\right)-U_{i=1}^{3} G_{i} \subseteq\left(R^{*}-\{\zeta\}\right)-u_{i=1}^{3} G_{i}
$$

For the reverse containment, if $\rho \varepsilon\left(R^{*}-\{\zeta\}\right)-U_{i=1}^{3} G_{i}$, then $\rho \varepsilon G_{1} \cup G_{2} \cup G_{3} \quad\{\zeta\}$ and there exists elements $\rho_{k}^{\prime}(1 \leq k \leq q)$ such that $\rho_{k}^{\prime} \varepsilon G_{1}$ for $k \leq r, \rho_{k}^{\prime} \varepsilon G_{2}$ for $r<k \leq p, p_{k}^{\prime} \in G_{3}$ for $p<k \leq q$, and such that

$$
\sum_{k=1}^{q} m_{k} /\left(\rho-\rho_{k}^{\prime}\right)=\left(\sum_{k=1}^{q} m_{k}\right) /(\rho-\zeta) .
$$

Therefore,

$$
\begin{aligned}
\left(\sum_{k=1}^{q} m_{k}\right) /(\zeta-\rho) & =\sum_{k=1}^{r} m_{k} /\left(\rho_{k}^{\prime}-\rho\right)+\sum_{k=r+1}^{p} m_{k} /\left(\rho_{k}^{\prime}-\rho\right)+\sum_{k=p+1}^{q} m_{k} /\left(\rho_{k}^{\prime}-\rho\right) \\
& =B_{1}+B_{2}+B_{3} \quad \text { (say). }
\end{aligned}
$$

Since $G_{1} \in D\left(K_{\omega}\right)$ and $\rho \notin G_{i}$ for $i=1,2,3$, we see from the definition of $g \cdot c \cdot r \cdot$ 's that $\phi_{\rho}\left(G_{i}\right)$ is $K_{0}$-convex for $i=1,2,3[5]$. In view of this and the fact that

$$
1 /\left(\rho_{k}^{\prime}-\rho\right)= \begin{cases}\phi_{\rho}\left(G_{1}\right) & \text { for } k=1,2, \ldots, r \\ \phi_{\rho}\left(G_{2}\right) & \text { for } k=r+1, \ldots, p \\ \phi_{\rho}\left(G_{3}\right) & \text { for } k=p+1, \ldots, q,\end{cases}
$$

we conclude that $B_{i} / A_{i} \varepsilon \phi_{\rho}\left(G_{i}\right)$ for $i=1,2,3$. Therefore, there exist elements $\rho_{i} \varepsilon G_{i}$ such that $B_{i} / A_{i}=1 /\left(\rho_{i}-\rho\right)$. Now, (4.9) implies (since $A_{1}+A_{2}+A_{3}=\sum_{k=1}^{q} m_{k}$ ) that 


$$
\sum_{i=1}^{3} A_{i} /\left(\rho-\rho_{i}\right)=\left(\sum_{i=1}^{3} A_{i}\right) /(\rho-\zeta),
$$

which says that $\rho \varepsilon\left(H^{*}-\{\zeta\}\right)-\bigcup_{i=1}^{3} G_{i}$. Hence

$$
\left(R^{*}-\{\zeta\}\right)-\bigcup_{i=1}^{3} G_{i} \subseteq\left(H^{*}-\{\zeta\}\right)-U_{i=1}^{3} G_{i} \cdot
$$

Finally (4.8) and (4.10) prove our lemma.

Next, we take up the most general theorem of this paper, which we establish via application of Theorem 3.1.

THEOREM 4.4. Let $M$ be a fully supportable subset of $V$ and, for $k=1,2, \ldots, q$, Let $\mathrm{P}_{\mathbf{k}} \in \mathbf{P}_{\mathrm{n}_{\mathrm{k}}}^{*}$ and $\mathrm{E}_{\mathrm{o}}^{(\mathrm{k})} \equiv \mathrm{E}_{\mathrm{o}}\left(\mathrm{N}, \mathrm{G}_{\mathrm{k}}\right)$ be circular cones in $\mathrm{E}$ such that $\mathrm{E}_{\mathrm{P}_{\mathbf{k}}}(\mathrm{x}, \mathrm{y}) \subseteq$ $\mathrm{T}_{\mathrm{G}_{\mathrm{k}}}(\mathrm{x}, \mathrm{y})$ for all $(\mathrm{x}, \mathrm{y}) \in \mathrm{N}$ and for all $\mathrm{k}$. If $\phi\left(\mathrm{Q} ; \mathrm{x}_{1}, \mathrm{x}\right)$ is the algebra-valued generalized polar of the product $Q(x)(\mathrm{cf} .(2.1)-(2.3))$ with $\mathrm{m}_{\mathrm{k}}>0$ for $\mathrm{k} \leq \mathrm{p}(\mathrm{p}<\mathrm{q})$ and $\mathrm{m}_{\mathrm{k}}<0$ for $\mathrm{k}>\mathrm{p}$, then $\Phi\left(\mathrm{Q} ; \mathrm{x}_{1}, \mathbf{x}\right) \in \mathrm{M}$ for all linearly independent elements $\mathbf{x}, \mathbf{x}_{1}$ of $\mathrm{E}$ such that $\mathrm{x}_{1} \varepsilon \mathrm{E}-\mathrm{n}_{\mathrm{k}=1}^{\mathrm{q}} \mathrm{E}_{\mathrm{o}}^{(\mathrm{k})}$ and $\mathrm{x} \in \mathrm{E}-\left(\underset{\mathrm{k}=1}{\mathrm{q}} \mathrm{E}_{\mathrm{o}}^{(\mathrm{k})}\right) \mathrm{u}$ $\mathrm{T}_{\mathrm{S}}\left(\mathrm{x}_{\mathrm{o}}, \mathrm{y}_{\mathrm{o}}\right)$, where $\mathrm{s}\left(\mathrm{x}_{\mathrm{o}}, \mathrm{y}_{\mathrm{o}}\right)$ is the set as defined in Theorem 3.1 .

PROOF. If $\xi \varepsilon V-M$, there is a unique nontrivial scalar homomorphism $L$ on $v$ such that $L(\xi)=0$ but $L(v) \neq 0$ for all $v \varepsilon M$. Now, $L P_{k} \in P_{n_{k}} \quad$ (2.4) and it can be easily shown that $\mathrm{Z}_{\mathrm{LP}_{k}}(x, y) \subseteq E_{P_{k}}(x, y) \subseteq T_{G_{k}}(x, y)$ for all $(x, y) \varepsilon N$ and for all k. In view of remark 2.1 and the discussion immediately preceding it (with the notations therein), we have

$$
L\left(Q\left(Q ; x_{1}, x\right)\right)=\$\left(L Q ; x_{1}, x\right),
$$

both sides using the same $\mathrm{m}_{k}{ }^{\prime} \mathrm{s}$. Applying Theorem 3.1 to the generalized polar $\Phi\left(L Q ; x_{1}, x\right)$ of the product $L Q$ of the polynomials $L P_{k}$, we see that $\$\left(L Q ; x_{1}, x\right) \neq 0$ for all linearly independent elements $x, x_{1}$ of $E$ as claimed. Consequently, the relations (4.11) implies that $\Phi\left(Q ; x_{1}, x\right) \neq \xi$ for all $x, x_{1}$ as claimed. Finally, the arbitrary nature of $\xi$ (in V-M) completes the proof.

The following version of Theorem 4.4 for the case when $\underset{k=1}{q} m_{k}=0$ and $\underset{k=1}{q} E_{0}^{(k)}=\phi$ is a result exclusively in terms of algebra-valued generalized polars with a vanishing weight. The proof is immediate as in the case of Theorem 3.2.

THEOREM 4.5. Under the notations and hypotheses of Theorem 4.4 if we assume that $\bigcap_{\mathrm{k}=1}^{\mathrm{q}} \mathrm{E}_{\mathrm{O}}^{(\mathrm{k})}=\phi$ and $\sum_{\mathrm{k}=1}^{\mathrm{q}} \mathrm{m}_{\mathrm{k}}=0$, then $\$\left(Q ; \mathrm{x}_{1}, \mathbf{x}\right) \in \mathrm{M}$ for all linearly independent $\mathrm{k}=1$
elements $\mathrm{x}, \mathrm{x}_{1}$ of $\mathrm{E}$ such that $\mathrm{x} \in \mathrm{E}-\mathrm{U}_{\mathrm{k}=1}^{\mathrm{q}+1} \mathrm{E}_{\mathrm{o}}^{(\mathrm{k})}$, where $\mathrm{E}_{\mathrm{o}}(\mathrm{q}+1) \equiv \mathrm{E}_{\mathrm{o}}\left(\mathrm{N}, \mathrm{G} \mathrm{q}_{\mathrm{q}}\right)$
is the cone as defined in Theorem 3.2. 
Since Theorem 4.4 reduces to Theorem 3.1 on taking $V=K$ and $M=K-\{0\}$ (c. $f$. Remark 4.1), it becomes the most general result of this paper. Besides, it leads to the following corollary, which combines two earlier results due to the authors [1] and [2] which includes in it (as a natural consequence) a number of other known results due to Zaheer [5], [17], Marden [3], Walsh [22], and to Bôcher [4] .

COROLLARY 4.6. Let $\mathrm{E}_{0, i} \equiv \mathrm{E}_{0}\left(\mathrm{~N}, G_{i}^{\prime}\right), i=1,2,3$, be circular cones in $\mathrm{E}$. Under the notations and hypotheses of Theorem 4.4, if the circular cones $\mathrm{E}_{\mathrm{o}}^{(\mathrm{k})} \equiv \mathrm{E}_{\mathrm{o}}\left(\mathrm{N}, \mathrm{G}_{\mathrm{k}}\right)$ are given by

$$
E_{0}^{(k)}=\left\{\begin{array}{lll}
E_{0,1} & \text { for } & 1 \leq k \leq r(r<p) \\
E_{0,2} & \text { for } & r<k \leq p \\
E_{0,3} & \text { for } & p<k \leq q,
\end{array}\right.
$$

then $\Phi\left(Q ; \mathbf{x}_{1}, \mathbf{x}\right) \in \mathbf{M}$ for all linearly independent elements $\mathbf{x}, \mathbf{x}$ of $\mathbf{E}$ such that $x_{1} \varepsilon E-\underset{i=1}{n^{3}} E_{0, i} \quad x \in E-\left(\underset{i=1}{u^{3}} E_{o, i}\right) \cup T_{S^{\prime}}\left(x_{0}, y_{0}\right)$, where $\left(x_{0}, y_{0}\right) \varepsilon N \cap L^{2}\left[x, x_{1}\right]$, $x_{1}=\gamma x_{0}+\delta y_{0}$ and

$$
S^{\prime}\left(x_{0}, y_{0}\right)=\left\{\begin{array}{llll}
\rho & \varepsilon & K_{\omega}
\end{array} \sum_{i=1}^{3} A_{i} /\left(\rho-\rho_{i}\right)=\left(\sum_{i=1}^{3} A_{i}\right) /(\rho-\gamma / \delta) ; \rho_{i} \varepsilon G_{i}^{\prime}\left(x_{0}, y_{0}\right)\right\},
$$

with

$$
A_{1}=\sum_{1=1}^{r} m_{k}, A_{2}=\sum_{k=r+1}^{p} m_{k} \text { and } A_{3}=\sum_{k=p+1}^{q} m_{k}
$$

PROOF. If $x, x_{1}$ are linearly independent elements of $E$ such that $x_{1} \notin n_{i=1}^{3} E_{0 . i}$

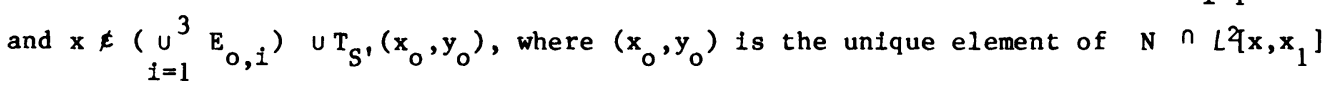
(see definition of $N$ ) then there exists a unique set of scalars $\alpha, \beta, \gamma, \delta$ (with $\alpha \delta-\beta \gamma \neq 0$ ) such that $x=\alpha x_{0}+\beta y_{0}, x_{1}=\gamma x_{0}+\delta y_{0}$ and

$$
\alpha / \beta \nsubseteq\left(u_{i=1}^{3} G_{i}^{\prime}\left(x_{0}, y_{0}\right)\right) \cup S^{\prime}\left(x_{0}, y_{0}\right) \quad(c f .(2.5)),
$$

where $S^{\prime}\left(x_{0}, y_{0}\right)=H^{*}$ for $\zeta=\gamma / \delta$ and $G_{i}=G_{i}^{\prime}\left(x_{0}, y_{0}\right)$ (cf. Lemma 4.3). Note that $\alpha \delta-\beta \gamma \neq 0$ implies that $\alpha / \beta \neq \gamma / \delta$. This implies that $\alpha / \beta \notin H^{*} U\{\gamma / \delta\} U$ $\left(u_{i=1}^{3} G_{i}^{\prime}\left(x_{0}, y_{0}\right)\right)$. Therefore,

$$
\alpha / \beta \notin\left(H^{*}-\{\gamma / \delta\}\right)-u_{1=1}^{3} G_{1}^{\prime}\left(x_{0}, y_{0}\right) \text {. }
$$

Since $x_{1} \notin \underset{i=1}{n^{3}} E_{0, i}$, we see that $\gamma / \delta \notin n_{i=1}^{3} G_{1}^{\prime}\left(x_{0}, y_{0}\right)$, where

$$
\mathrm{R}^{*}=\left[\left.\begin{array}{lll}
\rho & \varepsilon & \mathrm{K}_{\omega}
\end{array}\right|_{\mathrm{k}=1} ^{\mathrm{q}} \mathrm{m}_{\mathrm{k}} /\left(\rho-\rho_{\mathrm{k}}\right)=\left(\sum_{\mathrm{k}=1}^{\mathrm{q}} \mathrm{m}_{\mathrm{k}}\right) /(\rho-\gamma / \delta) ; \rho_{1}, \ldots, \rho_{\mathbf{r}} \varepsilon\right.
$$




$$
\left.G_{1}^{\prime}\left(x_{o}, y_{o}\right) ; \rho_{r+1}, \ldots, \rho_{p} \varepsilon G_{2}^{\prime}\left(x_{o}, y_{o}\right) ; \rho_{p+1}, \ldots, \rho_{q} \varepsilon G_{3}^{\prime}\left(x_{o}, y_{o}\right)\right\} .
$$

That is,

$$
\alpha / \beta \notin R^{*} \cup\{\gamma / \delta\} \cup\left(U_{i=1}^{3} G_{i}^{\prime}\left(x_{0}, y_{0}\right)\right)
$$

Consequently, $\alpha / \beta \notin R^{*}$. Since the $G_{k}$ of Theorem 4.4 in the present set up are given by

$$
G_{k}= \begin{cases}G_{1}^{\prime} & \text { fpr } 1 \leq k \leq r \\ G_{2}^{\prime} & \text { for } r<k \leq p \\ G_{3}^{\prime} & \text { fpr } p<k \leq q,\end{cases}
$$

we have that $R^{*}=S\left(x_{0}, y_{0}\right), \underset{k=1}{u^{q}} E_{0}^{(k)}=u_{i=1}^{u^{3}} E_{o, i}$ and ${ }_{i=1}^{q} E_{0}^{(k)}=\prod_{i=1}^{n} E_{0, i}$ (cf. (4.12)). Therefore, we see that $\alpha / \beta \notin \mathrm{s}\left(\mathrm{x}_{0}, \mathrm{y}_{0}\right)$. Consequently, $\mathrm{x}$ and $\mathrm{x}_{1}$ are linearly independent elements of $E$ such that $x_{1} \notin \underset{k-1}{n} E_{0}^{(k)}$ and $x \notin \underset{k=1}{q} E_{0}^{(k)}$ ) $\cup T_{S}\left(x_{0}, y_{0}\right)$. By Theorem 4.4, $P\left(Q ; x_{1}, x\right) \varepsilon M$, as was to be proved.

If $\sum_{k=1}^{q} m_{k} \neq 0$, Corollary $(4.6)$ is Theorem 4.3 of a paper due to the authors [2], and if (in addition) $V=K$ and $M=K-\{0\}$, it is Theorem 3.1 in the same paper. In case when $\sum_{k=1}^{q} m_{k}=0$ and $n_{i=1}^{3} E_{o, i}=\phi$, Corollary 4.6 leads to the following corollary, which is again a result due to the authors [1] and which reduces to Theorem 3.1 [1] when specialized for $V=K$ and $M=K-\{0\}$.

COROLLARY 4.7. Under the notations and hypotheses of Corollary 4.6 if $\sum_{k=1}^{q} m_{k}=0$ and $n_{i=1}^{3} \mathrm{E}_{\mathrm{O}, \mathrm{i}}=\phi$, then $\Phi\left(Q ; \mathrm{x}_{1}, \mathbf{x}\right) \in \mathrm{M}$ for all linearly independent elements $\mathbf{x}, \mathrm{x}_{1}$ of $\mathrm{E}$ such that $\mathrm{x} \in \mathrm{E}-\mathrm{U}_{\mathrm{i}=1}^{4} \mathrm{E}_{\mathrm{O}, \mathrm{i}}$, where $\mathrm{E}_{\mathrm{O}, 4} \equiv \mathrm{E}_{\mathrm{O}}\left(\mathrm{N}, \mathrm{G}_{4}^{\prime}\right)$ is the cone defined by

$$
G_{4}^{\prime}\left(x_{0}, y_{0}\right)=\left\{\rho \in K_{\omega} \mid\left(\rho, \rho_{3}, \rho_{2}, \rho_{1}\right)=-A_{2} / A_{1} ; \rho_{1} \varepsilon G_{i}^{\prime}\left(x_{0}, y_{0}\right)\right\}
$$

for all $\left(\mathrm{x}_{\mathrm{o}}, \mathrm{y}_{\mathrm{o}}\right) \in \mathrm{N}$, with $\mathrm{A}_{1}=\sum_{\mathrm{k}=1}^{\mathrm{r}} \mathrm{m}_{\mathrm{k}}$ and $\mathrm{A}_{2}=\sum_{\mathrm{k}=\mathrm{r}+1}^{\mathrm{p}} \mathrm{m}_{\mathrm{k}}$.

PROOF. If $x, x_{1}$ are linearly independent elements of $E$ such that $x \notin \cup_{i=1}^{4} E_{0, i}$, then there exist a unique element $\left(x_{0}, y_{0}\right) \in N \cap L^{2}\left[x, x_{1}\right]$ and a unique set of scalars $\alpha, \beta, \gamma, \delta \quad$ (with $\alpha \delta-\beta \gamma \neq 0$ ) such that $x=\alpha x_{0}+\beta y_{0}$ and $x_{1}=\gamma x_{0}+\delta y_{0}$. Then $\alpha / \beta \notin \bigcup_{i=1}^{4} G_{i}^{\prime}\left(x_{0}, y_{0}\right)$, where $G_{4}^{\prime}\left(x_{0}, y_{0}\right)=R_{\lambda}$ for $\lambda=A_{2} / A_{1}$ and $G_{i}=G_{i}^{\prime}\left(x_{0}, y_{0}\right)$ 
(Lemma 4.2). We divide the proof into the following two cases:

Case (i). $\alpha / \beta \neq \omega$. In this case

$$
\alpha / \beta \notin R_{\lambda} \cup\{w\} \quad \cup\left(U_{i=1}^{3} G_{i}^{\prime}\left(x_{0}, y_{0}\right)\right)
$$

and so $\alpha / \beta \notin\left(R_{\lambda}-\{\omega\}\right)-u_{i=1}^{3} G_{i}^{\prime}\left(x_{0}, y_{0}\right)$. Since $n_{i=1}^{3} G_{i}^{\prime}\left(x_{0}, y_{0}\right)=\phi$, Lemma 4.2 implies that $\alpha / \beta \notin\left(\mathrm{H}_{\lambda}-\{\omega\}\right)-U_{i=1}^{i=1} G_{i}^{\prime}\left(x_{0}, y_{0}\right)$, where (since $\lambda=A_{2} / A_{1}$ and $A_{1}+A_{2}+A_{3}=0$, where $\left.A_{3}=\sum_{k=p+1}^{q} m_{k}\right)$

$$
\begin{array}{rlrl}
H_{\lambda} & =\left\{\rho \in K_{\omega} \mid \sum_{i=1}^{3} A_{i} /\left(\rho-\rho_{i}\right)=0 ; \rho_{i} \varepsilon G_{i}^{\prime}\left(x_{o}, y_{0}\right)\right\} \\
& =s^{\prime}\left(x_{0}, y_{0}\right) & (c f . \text { Corollary }(4.6) .
\end{array}
$$

Therefore,

$$
\alpha / \beta \notin S^{\prime}\left(x_{0}, y_{0}\right) \cup\{\omega\} \cup\left(u_{i=1}^{3} G_{i}^{\prime}\left(x_{0}, y_{0}\right)\right)
$$

and (hence) $\alpha / \beta \notin S^{\prime}\left(x_{0}, y_{0}\right)$. That is, $x \notin T_{S^{-}}\left(x_{0}, y_{0}\right)$. Consequently, $x$ and $x_{1}$ are linearly independent elements of E such that $x_{1} \notin n_{i=1}^{3} E_{0, i}=\phi$ and $x \notin\left(u_{i=1}^{3} E_{0, i}\right) u$ $\cup \mathrm{T}_{\mathrm{S}^{\prime}}\left(\mathrm{x}_{\mathrm{o}}, \mathrm{y}_{\mathrm{O}}\right)$. Finally, Corollary 4.6 says that $\Phi\left(Q ; \mathrm{x}_{1}, \mathrm{x}\right) \varepsilon \mathrm{M}$. as was to be proved.

Case (ii). $\alpha / \beta=\omega$. In the case under consideration $\beta=0$ and

$$
\alpha / \beta=\omega \notin R_{\lambda} \cup\left(U_{i=1}^{3} G_{i}^{\prime}\left(x_{0}, y_{0}\right)\right)
$$

If $\xi \varepsilon V-M$, there exists a unique nontrivial scalar homomorphism $L$ on $V$ such that $L(\xi)=0$ but $L(v) \neq 0$ for $v \in M$. Since the hypotheses of Theorem 4.4 are satisfied for the choice of circular cones given by (4.12), we proceed as in the proof of Theorem 4.4 and again observe that the polynomials $L P_{k}\left(\equiv P_{k}^{*}\right.$, say) satisfy the hypotheses of Theorem 3.1 with the $E_{0}^{(k)}$ and the $G_{k}$ given by (4.12) and (4.13). The fact that $P_{k}^{*}$ is an a.h.p. of degree $n_{k}$ from $E$ to $K$ allows us to write it in the form

$$
P_{k}^{*}\left(s x+t x_{1}\right)=\prod_{j=1}^{n_{k}}\left(\delta_{j k} s-\gamma_{j k} t\right), k=1,2, \ldots, q
$$

Since $x$ is not in the set $U_{i=1}^{3} E_{0,1}={\underset{k=1}{U}}_{E_{0}}^{(k)}$ and since $Z_{P_{k}^{*}}\left(x_{0}, y_{0}\right) \subseteq T_{G_{k}}\left(x_{0}, y_{0}\right)$ for all $k$, we see that $P_{k}^{*}(x)=\pi_{j=1}^{n_{k}} \delta_{j k} \neq 0$ for all k. Now, proceeding exactly on the lines of proof of Theorem 3.1 (except that we replace $P_{k}$ by $P_{k}^{*}$ and take $\beta=0$ all along) and using the same notations, we find that there exist elements $\rho_{k} \in G_{k}\left(x_{0}, y_{0}\right)$ such that $\nu_{k}=m_{k}\left(\delta \rho_{k}-\gamma\right) / \alpha$. Note that all the $\nu_{k}$ 's cannot vanish simultaneously (since $\bigcap_{k=1}^{q} E_{0}^{(k)}=n_{1=1}^{3} E_{0,1}=\phi$ ). Now, 


$$
\begin{aligned}
\sum_{k=1}^{\mathrm{q}} \nu_{k} & =\delta / \alpha\left[\sum_{k=1}^{\mathrm{r}} \mathrm{m}_{k} \rho_{k}+\sum_{k=\mathrm{r}+1}^{\mathrm{p}} \mathrm{m}_{\mathrm{k}} \rho_{k}\right]+\sum_{\mathrm{k}=\mathrm{p}+1}^{\mathrm{q}} \mathrm{m}_{\mathrm{k}} \rho_{\mathrm{k}}\left(\text { since } \sum_{\mathrm{k}=1}^{\mathrm{q}} \mathrm{m}_{\mathrm{k}}=0\right) \\
& =(\delta / \alpha)\left[\mathrm{B}_{1}+\mathrm{B}_{2}+\mathrm{B}_{3}\right] \text {, say. }
\end{aligned}
$$

Since $G_{i}^{\prime}\left(x_{0}, y_{0}\right)$ are $k_{0}$-convex $(4.14)$, we conclude from (4.13) that $B_{i} / A_{i} \varepsilon G_{i}^{\prime}\left(x_{o}, y_{o}\right)$, where $A_{3}=\sum_{k=p+1}^{q} m_{k} \cdot$ Now, there must exist elements $\rho_{i}^{\prime} \varepsilon G_{i}^{\prime}\left(x_{0}, y_{0}\right)$ such that $B_{i}=A_{i} \rho_{i}^{\prime}$ for $i=1,2,3$, and (hence)

$$
\sum_{k=1}^{q} \nu_{k}=(\delta / \alpha)\left[A_{1}\left(\rho_{1}^{\prime}-\rho_{3}^{\prime}\right)+A_{2}\left(\rho_{2}^{\prime}-\rho_{3}^{\prime}\right)\right] \text {, }
$$

because $A_{1}+A_{2}+A_{3}=0$. From Remark 2.1 and equation (3.4) [5] we have

$$
L\left(\phi\left(Q ; x_{1}, x\right)\right)=\phi\left(L Q ; x_{1}, x\right)=-\left(\sum_{k=1}^{q} \nu_{k}\right) . \prod_{k=1}^{\Pi^{q}} P_{k}(x) \neq 0,
$$

where $L Q$ is the product of a.h.p.'s $\mathrm{P}_{k^{*}}^{*}$ Since $\mathrm{P}_{k}^{*}(x) \neq 0$ for all $k,(4.15)$ would imply that (since $\delta \neq 0$ )

$$
A_{1}\left(\rho_{1}^{\prime}-\rho_{3}^{\prime}\right)+A_{2}\left(\rho_{2}^{\prime}-\rho_{3}^{\prime}\right)=0
$$

Note that $\rho_{1}^{\prime}, \rho_{2}^{\prime}, \rho_{3}^{\prime}$ must be distinct elements of $K$ (since $A_{1}, A_{2}>0, n_{i=1}^{3}$ $G_{i}^{\prime}\left(x_{0}, y_{0}\right)=\phi$ and $(4.14)$ holds $)$. Therefore, $\left(\omega, \rho_{3}^{\prime}, \rho_{2}^{\prime}, \rho_{1}^{\prime}\right)=\left(\rho_{3}^{\prime}-\rho_{1}^{\prime}\right) /\left(\rho_{3}^{\prime}-\rho_{2}^{\prime}=\right.$ $-A_{2} / A_{1}=-\lambda$, and $s o \alpha / B=\omega \varepsilon R_{\lambda}$ This contradicts equation (4.14). Consequently, (4.16) holds and $\Phi\left(Q ; x_{1}, x\right) \neq \xi$ for any $\xi \varepsilon V-M$. That is, $\Phi\left(Q ; x_{1}, x\right) \varepsilon M$, as was to be proved.

Finally, cases (i) and (ii) complete the proof.

The following Corollaries 4.8 and 4.9 can be proved directly from Theorem 4.4, via applications of a suitably modified form of Lemmas 4.2 and 4.3 , exactly in the manner in which Corollaries 4.6 and 4.7 have been derived with the help of Lemmas 4.2 and 4.3. But it would neither be necessary nor worthwhile to do so. This is because it has already been proved in earlier papers due to the authors Corollary 4.5 [1] and Corollary 4.4 [2], that Corollary 4.8 (resp. Corollary 4.9) follows from Corollary 4.6 (resp. Corollary 4.7). We, therefore, state these without proof.

COROLLARY 4.8 [17]. Let $\mathrm{E}_{\mathrm{o}, i} \equiv \mathrm{E}_{\mathrm{o}}\left(\mathrm{N}, \mathrm{G}_{i}^{\prime}\right), i=1,2$, be circular cones in $\mathrm{E}$. Under the notations and hypotheses of Theorem 4.4, if $\sum_{\mathrm{k}=1}^{\mathrm{q}} \mathrm{m}_{\mathrm{k}} \neq 0$ and if the circular cones $\mathrm{E}_{\mathrm{o}}^{(\mathrm{k})} \equiv \mathrm{E}_{\mathrm{o}}\left(\mathrm{N}, \mathrm{G}_{\mathrm{k}}\right)$ are given by 


$$
E_{0}^{(k)}= \begin{cases}E_{0,1} & k=1,2, \ldots, p(p<q) \\ E_{0,2} & k=p+1, \ldots, q,\end{cases}
$$

then $\Phi\left(Q ; \mathbf{x}_{1}, \mathbf{x}\right) \in \mathbf{M}$ for all linearly independent elements $\mathbf{x}, \mathbf{x}_{1}$ of $\mathrm{E}$ such that

$\mathrm{x}_{1} \notin \mathrm{E}_{0,1} \cap \mathrm{E}_{0,2}$ and $\mathrm{x} \notin \mathrm{E}_{\mathrm{o}, 1} \cup \mathrm{E}_{0,2} \cup \mathrm{T}_{\mathrm{S}^{*}}\left(\mathrm{x}_{\mathrm{o}}, \mathrm{y}_{\mathrm{o}}\right)$, where $\left(\mathrm{x}_{\mathrm{o}}, \mathrm{y}_{\mathrm{o}}\right)$

$\varepsilon \mathrm{N} \cap L^{2}\left[\mathrm{x}, \mathrm{x}_{1}\right], \mathrm{x}_{1}=\gamma \mathrm{x}_{\mathrm{o}}+\delta \mathrm{x}_{\mathrm{o}}$ and

$$
S^{*}\left(\mathrm{x}_{0}, \mathrm{y}_{0}\right)=\left\{\rho \varepsilon \mathrm{K}_{\omega} \mid\left(\rho, \gamma / \delta, \rho_{1}, \rho_{2}\right)=-\mathrm{A}^{\prime} / \mathrm{A}^{\prime \prime} ; \rho_{i} \varepsilon \mathrm{G}_{i}^{\prime}\left(\mathrm{x}_{\mathrm{o}}, \mathrm{y}_{\mathrm{o}}\right)\right\},
$$

with $\mathrm{A}^{\prime}=\sum_{\mathrm{k}=1}^{\mathrm{p}} \mathrm{m}_{\mathrm{k}}$ and $\mathrm{A}^{\prime \prime}=\sum_{\mathrm{k}=\mathrm{p}+1}^{\mathrm{q}} \mathrm{m}_{\mathrm{k}}{ }^{\prime}$

COROLLARY 4.9 [17]. ${ }_{\mathrm{q}}$ Under the same notations and hypotheses as in Corollary 4.8, except that this time $\mathrm{k}_{\mathrm{k}} \mathrm{m}_{\mathrm{k}}=0$ and $\mathrm{E}_{0,1} \cap \mathrm{E}_{0,2}=\phi$, we have that

$\checkmark\left(Q ; x_{1}, \mathbf{x}\right) \in M$ for all linearly independent elements $\mathbf{x}, \mathrm{x}_{1}$ of $\mathrm{E}$ such that

$x \in E-E_{0,1} \cup E_{0,2}$.

For $\mathrm{V}-\mathrm{K}$ and $\mathrm{M}=\mathrm{K}-\{0\}$, the above Corollaries 4.8 and 4.9 are known results due to Zaheer [5].

At the end it emerges that Theorem 4.4 of this paper happens to be the most general result known thus far on (algebra-valued) generalized polars, whether having a vanishing or a nonvanishing weight, and it includes in it all the corresponding results that have been established earlier in the papers due to Marden [11], Zaheer [5], and to the authors [1] and [2]. It also includes improved versions of some we11known classical results, such as: Walsh's two-circle theorems [5], Marden's general theorem [7] expressed in Corollary 3.3, and Bocher's theorem [5]. To sum up: apart from the fact that all the previously known results [3], [5], [2], have been jacketted into Theorem 4.4, the present study answers in full generality the type of problem on generalized polar pursued since 1971, and, it unifies the hitherto unnecessary and separate treatments traditionally meted out to the cases of the vanishing and the nonvanishing weight. With Theorem $4.4 \mathrm{in}$ view, it may be pointed out there is no scope left for further studies in this subject area, except possibly when different new concepts are developed on some other lines.

ACXNOWLEDGMENT: This paper was supported by the Research Center Project No. Math/1406/29 of the Faculty of Science, King Saud University, Riyadh, Saudi Arabia.

\section{REFERENCES}

1. ZAHEER, N. and KHAN, A.A. Cross-ratio Theorems on Generalized Polars of Abstract homogeneous polynomials, Ann. di Mat. (IV) EXXVI (1980), 363-377.

2. ZAHEER, N. and KHAN, A.A. Some Theorems on Generalized Polars With a Nonvanishing Weight EYEUOEPIA, J. Math. Seminar Univ. of Athens, Greece, to appear.

3. MARDEN, M. A Generalization of a Theorem of Bocher, SIAM J. Numer. Ana1. 3 (1966) , 269-275. 
4. BôCHER, M. A Problem in Statics and its Relation to Certain Algebraic Invariants, Proc. Amer. Acad. Arts Sci. 40 (1904), 469-484.

5. ZAHEER, N. On Generalized Polars of the Product of Abstract Homogeneous Polynomials, Pacific J. Math., 74 No. 2 (1978), 535-557.

6. HORMANDER, L. On a Theorem of Grace, Math. Scand. 2 (1954), 55-64.

7. MARDEN, M. Geometry of Polynomials, rev. ed., Math. Surveys, No. 3, Amer. Math. Soc., Providence, RI, 1966.

8. MARDEN, M. On Composite Abstract Homogeneous Polynomials, Proc. Amer. Math. Soc. $\underline{22}$ (1969), 28-38.

9. ZAHEER, N. Null-sets of Abstract Homogeneous Polynomials in Vector Spaces, Doctoral Thesis, Univ. of Wisconsin, Milwaukee, WI, 1971.

10. ZAHEER, N. On Polar Relations of Abstract Homogeneous Polynomials, Trans. Amer. Math Soc. 218 (1976), 115-131.

11. ZAHEER, N. On Composite Abstract Homogeneous Polynomials, Trans. Amer. Math. Soc. 228 (1977), 345-358.

12. ZAHEER, N. and ALAM, M. Zeros of Polar-composite Polynomials in Algebraically Closed Fields, Proc. Lond. Math. Soc. (3) 40 (1980), 527-552

13. ZAHEER, N. and ALAM, M. Some Applications of the Theory of Polar-composite Polynomials, J. Lond. Math. Soc. (2) 22 (1980), 403-410.

14. ALAM, M. Composite Polynomials in Algebraica1ly Closed Fields, Doctoral Thesis, Aligarh Musilm Univesity, Aligarh, India, 1977.

15. HILLE, E. and PHILLIPS, R.S. Functional Analysis and Semi-groups, rev. ed., Amer. Math. Soc. Colloq. Pub1., Vol. 31, Amer. Math. Soc., Providence, RI, 1957.

16. TAYLOR, A.E. Additions to the Theory of Polynomials in Normed Linear Spaces, Tohoku Math. J. 44 (1938), 302-318.

17. ZAHEER, N. On the Theory of Algebra-valued Generalized Polars, Indiana Univ. Math. J. 29 (1980), No. 5, 693-701.

18. WILANSKY, A. Functional Analysis, Blaisde11, New York, 1964.

19. BOURBAKI, N. Éléments de Mathématique. XIV. Part 1, Les Structures Fondamentales de l'analyse. Livre II: Algèra. Chap. VI: Groupes et Corps ordonnés, Actualities Sci. Indust., 1179 , Hermann, Paris, 1952 .

20. VAN DER WAERDEN, B.L. Algebra, Vol. I, 4 th ed., Die Grundlehren der Math. Wissenschaften, Band 33, Springer-Verlag, Berlin, 1955, Eng1ish Trans. Ungar, New York, 1970.

21. ZERVOS, S.P. Aspects Modernes de 1a Localisation des Zeros des Polynomes d'unes Variable, Ann. Sci. Ecole Norm. Sup. (3) 77 (1960), 303-410.

22. WALSH, J.L. On the Location of the Roots of the Jacobian of Two Binary Forms, and of the Derivative of a Rational Function, Trans. Amer. Math. Soc. 22 (1921), 101-116.

23. MARDEN, M. On the Zeros of Linear Partial Fractions, Trans. Amer. Math. Soc. 32 (1930), 81-109.

24. MARDEN, $M$. On the Zeros of the Derivative of a Rational Function, Bu11. Amer. Math. Soc. 42 (1936), 400-405. 


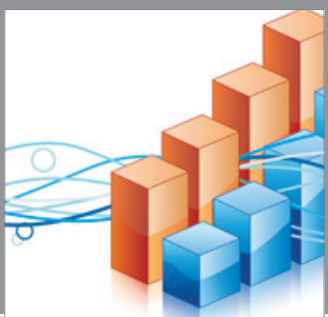

Advances in

Operations Research

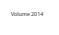

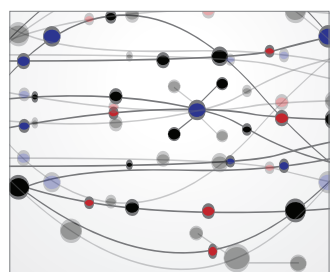

\section{The Scientific} World Journal
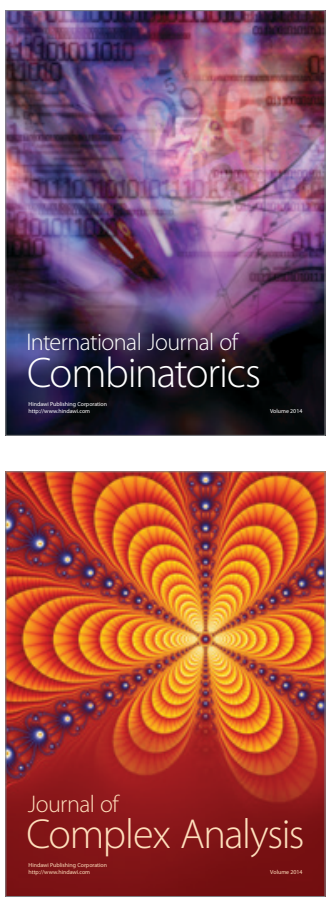

International Journal of

Mathematics and

Mathematical

Sciences
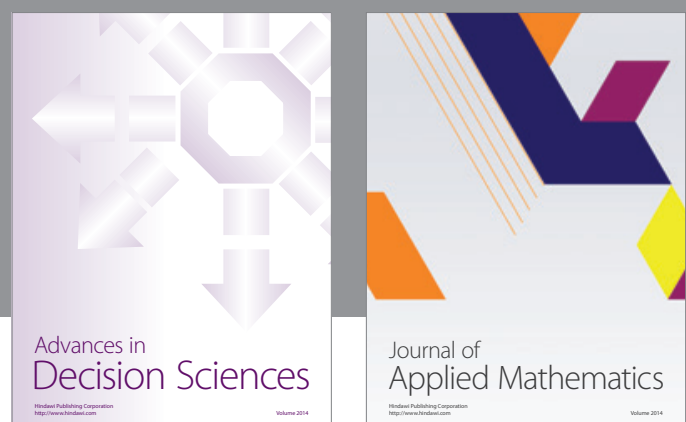

Journal of

Applied Mathematics
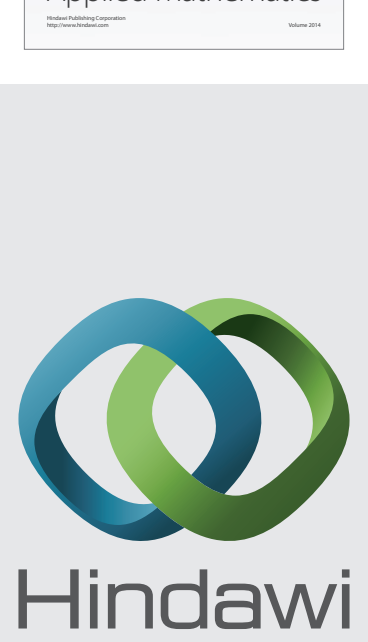

Submit your manuscripts at http://www.hindawi.com
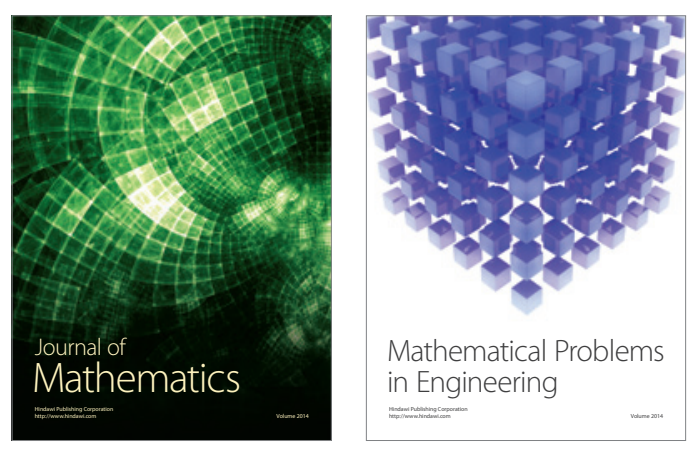

Mathematical Problems in Engineering
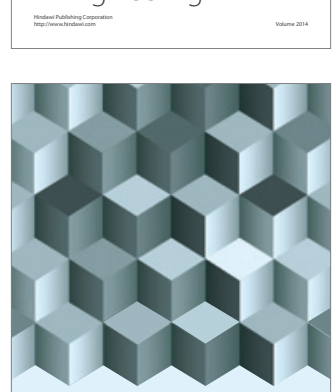

Journal of

Function Spaces
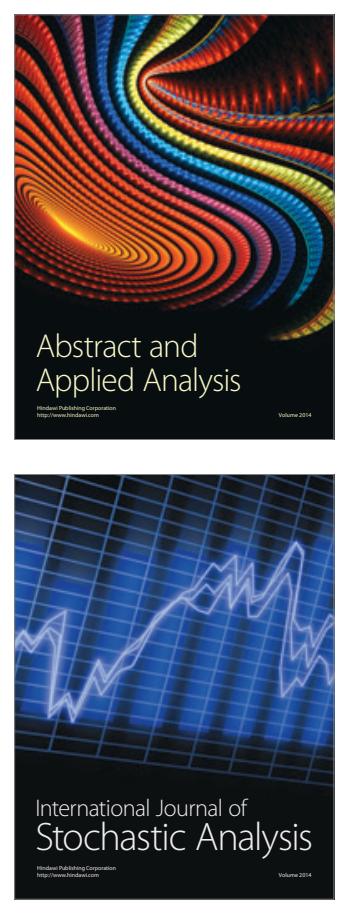

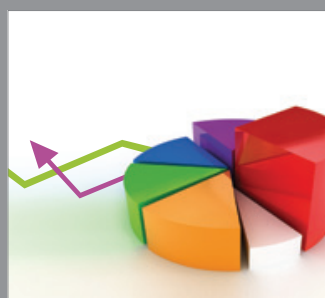

ournal of

Probability and Statistics

Promensencen
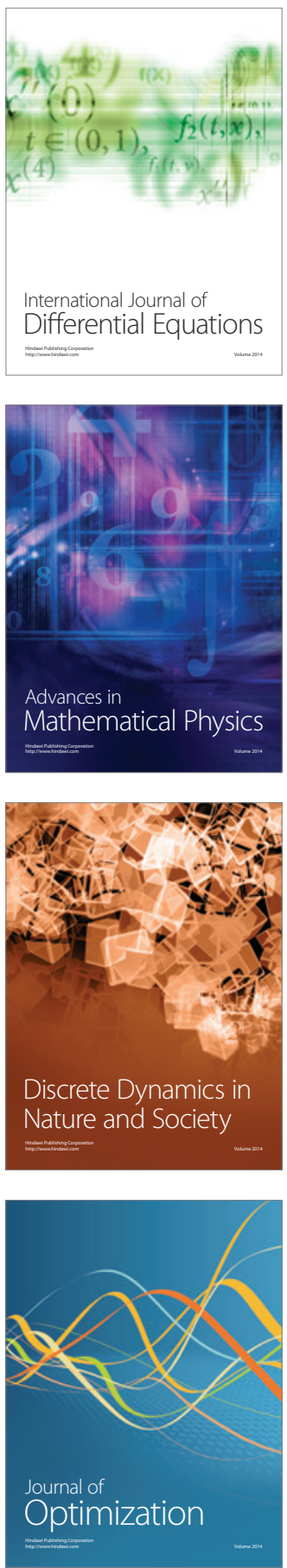